\title{
Symmetries of the Dirac operators associated with covariantly constant Killing-Yano tensors
}

\author{
Ion I. Cotăescu * \\ West University of Timişoara, \\ V. Pârvan Ave. 4, RO-1900 Timişoara, Romania \\ Mihai Visinescu ${ }^{\dagger}$ \\ Department of Theoretical Physics, \\ National Institute for Physics and Nuclear Engineering, \\ P.O.Box M.G.-6, Magurele, Bucharest, Romania
}

\begin{abstract}
The continuous and discrete symmetries of the Dirac-type operators produced by particular Killing-Yano tensors are studied in manifolds of arbitrary dimensions. The Killing-Yano tensors considered are covariantly constant and realize certain square roots of the metric tensor. Such a Killing-Yano tensor produces simultaneously a Dirac-type operator and the generator of a one-parameter Lie group connecting this operator with the standard Dirac one. The Dirac operators are related among themselves through continuous or discrete transformations. It is shown that the groups of the continuous symmetry can be only $U(1)$ and $S U(2)$, specific to (hyper-)Kähler spaces, but arising even in cases when the requirements for these special geometries are not fulfilled. The discrete symmetries are also studied obtaining the discrete groups $\mathbb{Z}_{4}$ and $\mathbb{Q}$. The briefly presented examples are the Euclidean Taub-NUT space and the Minkowski spacetime.
\end{abstract}

Pacs 04.62.+v

Key words: Dirac-type operators, Killing-Yano tensors, symmetries, supersymmetries.

\footnotetext{
*E-mail: cota@physics.uvt.ro

${ }^{\dagger}$ E-mail: mvisin@theor1.theory.nipne.ro
} 


\section{Introduction}

The (skew-symmetric) Killing-Yano (K-Y) tensors, that were first introduced by Yano [1] from purely mathematical reasons, are profoundly connected to the supersymmetric classical and quantum mechanics on curved manifolds where such tensors do exist 2. The K-Y tensors play an important role in theories with spin and especially in the Dirac theory on curved spacetimes where they produce first order differential operators, called Dirac-type operators, which anticommute with the standard Dirac one, $D$ [3]. Another virtue of the $\mathrm{K}-\mathrm{Y}$ tensors is that they enter as square roots in the structure of several second rank Stäckel-Killing tensors that generate conserved quantities in classical mechanics or conserved operators which commute with $D$. The construction of Carter and McLenaghan depended upon the remarkable fact that the (symmetric) Stäckel-Killing tensor $K_{\mu \nu}$ involved in the constant of motion quadratic in the four-momentum $p_{\mu}$

$$
Z=\frac{1}{2} K^{\mu \nu} p_{\mu} p_{\nu}
$$

has a certain square root in terms of K-Y tensors $f_{\mu \nu}[4]$ :

$$
K_{\mu \nu}=f_{\mu \lambda} f_{\cdot \nu}^{\lambda}
$$

The K-Y tensor here is a 2 -form $f_{\mu \nu}=-f_{\nu \mu}$ which satisfies the equation

$$
f_{\mu \nu ; \lambda}+f_{\mu \lambda ; \nu}=0 .
$$

These attributes of the K-Y tensors lead to an efficient mechanism of supersymmetry especially when the Stäckel-Killing tensor $K_{\mu \nu}$ in Eq. (11) is proportional with the metric tensor $g_{\mu \nu}$ and the corresponding K-Y tensors in Eq. (2) are covariantly constant. Then each tensor of this type, $f^{i}$, gives rise to a Dirac-type operator, $D^{i}$, representing a supercharge of the superalgebra $\left\{D^{i}, D^{j}\right\} \propto D^{2} \delta_{i j}$.

The typical example is the Euclidean Taub-NUT space which is a hyperKähler manifold possessing three covariantly constant K-Y tensors with realvalued components which constitute a hypercomplex structure generating a $N=4$ superalgebra at the level of the Dirac theory [5]. Moreover, each involved $\mathrm{K}-\mathrm{Y}$ tensor is a root of the metric tensor as it results from the definition of the Kählerian geometries (given in Appendix A). It is worth pointing out that the Euclidean Taub-NUT space has, in addition, a non-covariantly 
constant K-Y tensor related to its specific hidden symmetry showed off by the existence of a conserved Runge-Lenz operator that can be constructed with the help of the Dirac-type operators produced by all the four K-Y tensors of this space [6].

In what concerns the superalgebras of Dirac-type operators, the inverse problem is to find the suitable conjectures allowing the construction of Diractype operators $D^{\prime}$ which should satisfy the condition $\left(D^{\prime}\right)^{2} \propto D^{2}$. It was shown that these can be produced by covariantly constant K-Y tensors having not only real-valued components but also complex ones [7, 8. This extension seems to be productive since it permits to construct superalgebras in the Dirac theory in Minkowski spacetime which is not Kählerian, having only complex-valued covariantly constant K-Y tensors [8]. For this reason, in what follows we shall consider such more general tensors, called roots (instead of complex structures) since all of them are, up to constants, roots of the metric tensor. We note that the complex structures defining Kählerian geometries are particular automorphisms of the tangent bundle while the roots we use here are automorphisms of the complexified tangent bundle.

It is known that in four-dimensional manifolds the standard Dirac operator and the Dirac-type ones can be related among themselves through continuous or discrete transformations [9, 8]. It is interesting that there are only two possibilities, namely either transformations of the $U(1)$ group associated with the discrete group $\mathbb{Z}_{4}$ or $S U(2)$ transformations and discrete ones of the quaternionic group $\mathbb{Q}[9,8]$. Particularly, in the case when the roots are real-valued (complex structures) the first type of symmetry is proper to Kähler manifolds while the second largest one is characteristic for hyperKähler geometries [9]. The problem is what happens in the case of manifolds with larger number of dimensions allowing complex-valued roots. Could we expect to find new larger symmetries? The present article is devoted to this problem.

Our main purpose is to investigate the specific symmetries of the Dirac operators constructed using roots in geometries of arbitrary dimensions. We start with the observation that any root give rise simultaneously to a Diractype operator and a generator of the one-parameter Lie group relating this operator to the standard Dirac one. In fact the group generator is the main piece of the theory since it is able to produce itself the Dirac-type operator through a simple commutation with the standard Dirac operator. Exploiting this mechanism we study the continuous and discrete symmetries of the Dirac-type operators showing that, as in the case of the Kählerian geome- 
tries, there exists only two types of continuous symmetries, $U(1)$ and $S U(2)$, and the corresponding discrete symmetries, $\mathbb{Z}_{4}$ and $\mathbb{Q}$ respectively. One of our important results is the concrete form of the $S U(2)$ transformations in the general case of any $4 n$-dimensional manifold equipped with sets of roots having similar algebraic properties as the quaternion units.

The paper is organized as follows. We start in the second section with the construction of a simple version of the Dirac theory in any dimensions defining the Dirac adjoint and the behavior of the Dirac matrices under transformations of the universal covering group of the gauge group or of the corresponding complexified groups. The next section is devoted to the main differential operators we use while in Sec.4 we define the roots and the Diractype operators discussing their main properties. The continuous symmetries of the Dirac-type operators are studied in Sec. 5 and 6 where we establish the form of the $U(1)$ and respectively $S U(2)$ transformations among the standard Dirac operator and the Dirac-type ones. Therein we show that other kind of symmetries are not allowed because of the restrictions imposed by the Frobenius theorem. The mentioned discrete symmetries are studied in Sec. 7 and finally we present our conclusions and comments. In Appendices we give the usual definition of the Kählerian geometries and discuss the fourdimensional examples of physical interest, the Euclidean Taub-NUT space and the Minkowski spacetime.

\section{Dirac spinors in any dimensions}

The theory of the Dirac spinors in arbitrary dimensions depends on the choice of the manifold and the Clifford algebra. Bearing in mind that the irreducible representations of the Clifford algebra can have only an odd number of dimensions, we start with a $2 l+1$-dimensional pseudo-Riemannian manifold $M_{2 l+1}$ whose flat metric $\tilde{\eta}$ (of its pseudo-Euclidean model) has the signature $\left(m_{+}, m_{-}\right)$where $m_{+}+m_{-}=m=2 l+1$. This is the maximal manifold that can be associated to the Clifford algebra [10] acting on the $2^{l}$-dimensional space $\Psi^{l}$ of the complex spinors $\psi=\varphi_{1} \otimes \varphi_{2} \ldots \otimes \varphi_{l}$ built using complex twodimensional Pauli spinors $\varphi$. In this algebra we take the standard Euclidean basis formed by the hermitian matrices $\tilde{\gamma}^{A}=\left(\tilde{\gamma}^{A}\right)^{+}(A, B, \ldots=1,2, \ldots, m)$ that obey $\left\{\tilde{\gamma}^{A}, \tilde{\gamma}^{B}\right\}=2 \delta^{A B} I$, and define the basis corresponding to the met- 
$\operatorname{ric} \tilde{\eta}$ as

$$
\gamma^{A}= \begin{cases}\tilde{\gamma}^{A} & \text { for } \quad A=1,2, \ldots, m_{+} \\ i \tilde{\gamma}^{A} & \text { for } \quad A=m_{+}+1, m_{+}+2, \ldots, m\end{cases}
$$

such that

$$
\left\{\gamma^{A}, \gamma^{B}\right\}=2 \tilde{\eta}^{A B} I
$$

Since the first $m_{+}$matrices $\gamma^{A}$ remain hermitian while the $m_{-}$last ones become anti-hermitian, it seems that the unitaryness of the theory is broken. However, this can be restored with the help of the generalized Dirac adjoint, $\bar{\psi}=\psi^{+} \gamma$, involving a hermitian matrix $\gamma=\gamma^{+}$which should play the role of a metric operator obeying the condition $(\gamma)^{2}=I$ and giving the Dirac adjoint of any matrix $X$ as $\bar{X}=\gamma X^{+} \gamma$. It is not difficult to show that the matrix $\gamma=\epsilon \gamma^{1} \gamma^{2} \ldots \gamma^{m_{+}}$is convenient for this role [11] if we choose the following values for the phase factor:

$$
\epsilon=\left\{\begin{array}{lll}
(i)^{\frac{m_{+}-1}{2}} & \text { for odd } & m_{+}<m \\
(i)^{\frac{m_{+}}{2}} & \text { for even } & m_{+}<m
\end{array} .\right.
$$

In the special case of the Euclidean metric (when $m_{-}=0$ ) we have the trivial solution $\gamma=I$. The algebraic properties of the matrix $\gamma$ depends on $m_{+}$such that for $m_{+}$taking odd values we have the following superalgebra

$$
\begin{aligned}
{\left[\gamma, \gamma^{A}\right]=0 } & \text { for } \quad A=1,2, \ldots, m_{+}, \\
\left\{\gamma, \gamma^{A}\right\}=0 & \text { for } \quad A=m_{+}+1, \ldots, m,
\end{aligned}
$$

while for even $m_{+}$the situation is reversed. Consequently, one can verify that

$$
\overline{\gamma^{A}}=\left\{\begin{array}{lll}
\gamma^{A} & \text { for odd } & m_{+} \\
-\gamma^{A} & \text { for even } & m_{+}
\end{array}, \quad A=1,2, \ldots, m,\right.
$$

which means that from the point of view of the Dirac adjoint all the matrices $\gamma^{A}$ have the same behavior, being either self-adjoint or anti self-adjoint. Thus the unitaryness of the theory is guaranteed. In what follows we consider that $m_{+}$is odd and match all the phase factors according to self-adjoint gamma matrices. When these are anti self-adjoint (because of an even $m_{+}$) it suffices to change $\gamma^{A} \rightarrow i \gamma^{A}$. Particularly, in the examples we give in different fourdimensional manifolds immersed in that of the Kaluza-Klein theory, we take all the space-like dimensions of negative signature.

The gauge group $G(\tilde{\eta})=O\left(m_{+}, m_{-}\right)$of the metric $\tilde{\eta}$, with the mentioned signature, admits an universal covering group $\mathcal{G}(\tilde{\eta})$ that is simply connected 
and has the same Lie algebra. In order to avoid complications due to the presence of these two groups we consider here that the gauge group is a vector representation of $\mathcal{G}(\tilde{\eta})$ and we denote by $G(\tilde{\eta})=\lambda[\mathcal{G}(\tilde{\eta})]$ all the equivalent vector representations. The spinor representation of the same group, denoted by $\sigma[\mathcal{G}(\tilde{\eta})]$, is carried by the space $\Psi^{l}$ being generated by the spin operators

$$
S^{A B}=\frac{i}{4}\left[\gamma^{A}, \gamma^{B}\right]
$$

which are self-adjoint, $\overline{S^{A B}}=S^{A B}$, and satisfy

$$
\begin{aligned}
{\left[S^{A B}, \gamma^{C}\right] } & =i\left(\tilde{\eta}^{B C} \gamma^{A}-\tilde{\eta}^{A C} \gamma^{B}\right) \\
{\left[S_{A B}, S_{C D}\right] } & =i\left(\tilde{\eta}_{A D} S_{B C}-\tilde{\eta}_{A C} S_{B D}+\tilde{\eta}_{B C} S_{A D}-\tilde{\eta}_{B D} S_{A C}\right) .
\end{aligned}
$$

as it results from Eqs. (5) and (9). It is known that for any real or complex valued skew-symmetric tensor $\omega_{A B}=-\omega_{B A}$ the operator

$$
T(\omega)=e^{i S(\omega)}, \quad S(\omega)=\frac{1}{2} \omega_{A B} S^{A B}
$$

transforms the gamma-matrices according to the rule

$$
T(\omega) \gamma^{A}[T(\omega)]^{-1}=\Lambda_{\cdot B}^{A \cdot}(\omega) \gamma^{B}
$$

where

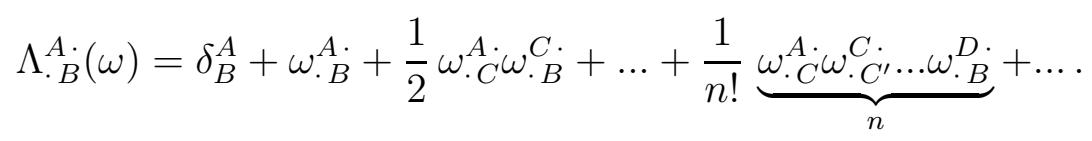

If $\omega \in \mathbb{R}$ then $\overline{S(\omega)}=S(\omega)$, the operators $T(\omega)$ are unitary with respect to the Dirac adjoint satisfying $\overline{T(\omega)}=[T(\omega)]^{-1}$ and, therefore, $T(\omega) \in \sigma[\mathcal{G}(\tilde{\eta})]$ and $\Lambda(\omega) \in \lambda[\mathcal{G}(\tilde{\eta})]$. However, for $\omega \in \mathbb{C}$ we have to consider the complexified group of $\mathcal{G}$, denoted by $\mathcal{G}_{c}$, and the corresponding vector and spinor representations.

\section{Differential operators}

In many physical problems involving differential operator these are defined on a domain of a manifold which, in general, may be a submanifold of $M_{m}$, $m=2 l+1$, like in the case of the $(1+3)$-dimensional spacetimes immersed 
in the five-dimensional manifold of the Kaluza-Klein theory where $l=2$. Therefore, it is indicated to consider the differential operators on a submanifold $M_{n} \subset M_{m}$ of dimension $n \leq m$ whose flat metric $\eta$ is a part (or restriction) of the metric $\tilde{\eta}$, having the signature $\left(n_{+}, n_{-}\right)$, with $n_{+} \leq m_{+}$, $n_{-} \leq m_{-}$and $n_{+}+n_{-}=n$. In $M_{n}$ we choose a local chart (i.e. natural frame) with coordinates $x^{\mu}, \alpha, \ldots, \mu, \nu, \ldots=1,2, \ldots, n$, and introduce local orthogonal non-holonomic frames using " $n$-beins", $e(x)$ and $\hat{e}(x)$, whose components are labeled by local (hated) indices, $\hat{\alpha}, \ldots \hat{\mu}, \hat{\nu}, \ldots=1,2, \ldots, n$, that represent a subset of the Latin capital ones, eventually renumbered. The local indices have to be raised or lowered by the metric $\eta$. The fields $e$ and $\hat{e}$ accomplish the conditions $e_{\hat{\alpha}}^{\mu} \hat{e}_{\nu}^{\hat{\alpha}}=\delta_{\mu}^{\nu}, e_{\hat{\alpha}}^{\mu} \hat{e}_{\mu}^{\hat{\beta}}=\delta_{\hat{\alpha}}^{\hat{\beta}}$ and orthogonality relations as $g_{\mu \nu} e_{\hat{\alpha}}^{\mu} e_{\hat{\beta}}^{\nu}=\eta_{\hat{\alpha} \hat{\beta}}$. With their help the metric tensor of $M_{n}$ can be put in the form $g_{\mu \nu}(x)=\eta_{\hat{\alpha} \hat{\beta}} \hat{e}_{\mu}^{\hat{\alpha}}(x) \hat{e}_{\nu}^{\hat{\beta}}(x)$. The spin connection $\Gamma_{\mu}=\frac{i}{2} e_{\hat{\nu}}^{\beta}\left(\hat{e}_{\alpha}^{\hat{\sigma}} \Gamma_{\beta \mu}^{\alpha}-\hat{e}_{\beta, \mu}^{\hat{\sigma}}\right) S_{\cdot}^{\hat{\nu}} \cdot \hat{\sigma}$ gives the action of the covariant derivatives in the spinor sector, $\nabla_{\mu} \psi=\left(\tilde{\nabla}_{\mu}+\Gamma_{\mu}\right) \psi$, where $\tilde{\nabla}_{\mu}$ is the usual covariant derivative (acting in natural indices). This definition is in accordance with the presented generalized theory of spinors since we have $\bar{\Gamma}_{\mu}=-\Gamma_{\mu}$ such that the quantity $\bar{\psi} \psi$ can be derived as a scalar, i.e. $\nabla_{\mu}(\bar{\psi} \psi)=\overline{\nabla_{\mu} \psi} \psi+\bar{\psi} \nabla_{\mu} \psi=\partial_{\mu}(\bar{\psi} \psi)$, while the quantities $\bar{\psi} \gamma^{\alpha} \gamma^{\beta} \ldots \psi$ behave as tensors of different ranks. Moreover, the use of covariant derivatives assures the covariance of the whole theory under the gauge transformations, $\hat{e}_{\mu}^{\hat{\alpha}}(x) \rightarrow \hat{e}_{\mu}^{\prime \hat{\alpha}}(x)=\Lambda_{\cdot \hat{\beta}}^{\hat{\alpha} \cdot}[\omega(x)] \hat{e}_{\mu}^{\hat{\beta}}(x)$, $e_{\hat{\alpha}}^{\mu}(x) \rightarrow e_{\hat{\alpha}}^{\prime \mu}(x)=\Lambda_{\hat{\alpha}}^{\cdot \hat{\beta}} \cdot[\omega(x)] e_{\hat{\beta}}^{\mu}(x)$ and $\psi(x) \rightarrow \psi^{\prime}(x)=\bar{T}[\omega(x)] \psi(x)$ due to $\Lambda[\omega(x)] \in \lambda[\mathcal{G}(\eta)]$ and $T[\omega(x)] \in \sigma[\mathcal{G}(\eta)]$ where $\omega_{\hat{\mu} \hat{\nu}}=-\omega_{\hat{\nu} \hat{\mu}}$ are real functions on $M$. We specify that these transformations can not be extended for $\omega(x)$ taking complex values since $e$ and $\hat{e}$ must remain real fields.

Thus we reproduced the main features of the familiar tetrad gauge covariant theories with spin in $(1+3)$-dimensions from which we can take over now all the results arising from similar formulas. In this way we find that the point-dependent matrices $\gamma^{\mu}(x)=e_{\hat{\alpha}}^{\mu}(x) \gamma^{\hat{\alpha}}$ and $S^{\mu \nu}(x)=e_{\hat{\alpha}}^{\mu}(x) e_{\hat{\beta}}^{\nu}(x) S^{\hat{\alpha} \hat{\beta}}$ have the same properties as (5), (9), (10) and (11), but with $g(x)$ instead of the flat metric, and recover the useful formulas

$$
\begin{aligned}
& \nabla_{\mu}\left(\gamma^{\nu} \psi\right)=\gamma^{\nu} \nabla_{\mu} \psi, \\
& {\left[\nabla_{\mu}, \nabla_{\nu}\right] \psi=\frac{1}{4} R_{\alpha \beta \mu \nu} \gamma^{\alpha} \gamma^{\beta} \psi}
\end{aligned}
$$

where $R$ is the Riemannian-Christoffel curvature tensor of $M_{n}$.

The operators we study here are involving only first order covariant 
derivatives and the natural objects of the theory like the Killing vectors or K-Y tensors. The simplest one is the standard Dirac operator

$$
D=i \gamma^{\alpha} \nabla_{\alpha}
$$

that is self-adjoint, $\bar{D}=D$, and covariantly transforms under gauge transformations, i.e. $D \rightarrow D^{\prime}=\bar{T} D T$. Other operators can be constructed using Killing vectors $\tilde{k}_{\mu}$ (that obey $\tilde{k}_{\mu ; \nu}+\tilde{k}_{\nu ; \mu}=0$ ) generalizing the result of Carter and McLenaghan 3 obtained for the Dirac fermions. Thus one can show that for any isometry of $M_{n}$ of Killing vector $\tilde{k}^{\mu}$ there is an appropriate operator

$$
X_{\tilde{k}}=i\left(\tilde{k}^{\mu} \nabla_{\mu}-\frac{1}{4} \gamma^{\mu} \gamma^{\nu} \tilde{k}_{\mu ; \nu}\right)
$$

which commutes with the standard Dirac operator. In addition, following the arguments of Ref. [12] one finds that $X_{v}$ is just a generator of the representation of the universal covering group of the isometry group of $M_{n}$ induced by $\sigma[\mathcal{G}(\eta)]$. Another result of Refs. [3, 13, 14] which holds in the $n$-dimensional case is that each $\mathrm{K}-\mathrm{Y}$ tensor $\tilde{f}$ of rank 2 produces the nonstandard Dirac operator of the form

$$
D_{\tilde{f}}=i \gamma^{\mu}\left(\tilde{f}_{\mu}^{\cdot \nu} \nabla_{\nu}-\frac{1}{6} \gamma^{\nu} \gamma^{\rho} \tilde{f}_{\mu \nu ; \rho}\right)
$$

which anticommutes with $D$. The operators $D_{\tilde{f}}$ will be called Dirac-type operators. In the following we shall focus on the properties of these operators and other ones related to K-Y tensors.

\section{Roots and Dirac-type operators}

Given $\xi$ an arbitrary tensor field of rank 2 defined on a domain of $M_{n}$, we denote with the same symbol $\langle\xi\rangle$ the equivalent matrices with the elements $\xi_{\cdot \nu}^{\mu}$ in natural frames as well as those having the matrix elements $\xi_{\cdot \hat{\beta}}^{\hat{\alpha}}=$ $\hat{e}_{\mu}^{\hat{\alpha}} \xi_{\nu}^{\mu} \cdot e_{\hat{\beta}}^{\nu}$ in local frames. We say that $\xi$ is non-singular on $M_{n}$ if $\operatorname{det}\langle\xi\rangle \neq 0$ on a domain of $M_{n}$ where the metric is non-singular. This tensor is said irreducible on $M_{n}$ if its matrix is irreducible.

Definition 1 The non-singular real or complex-valued $K-Y$ tensor $f$ of rank 2 defined on $M_{n}$ which satisfies

$$
f_{\cdot \alpha}^{\mu \cdot} f_{\mu \beta}=g_{\alpha \beta}
$$


is called an unit root of the metric tensor of $M_{n}$, or simply an unit root of $M_{n}$.

Any K-Y tensor that satisfy Eq. (201) is covariantly constant [7, i.e.,

$$
f_{\mu \nu ; \sigma}=0 .
$$

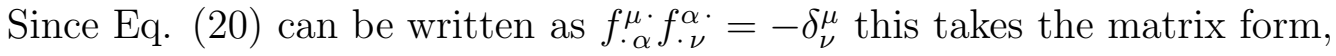

$$
\langle f\rangle^{2}=-\mathbf{I},
$$

where the notation $\mathbf{I}$ stands for the $n \times n$ identity matrix. Hereby we see that the unit roots are matrix representations of several complex units (similar to $i \in \mathbb{C}$ ) with usual properties as, for example, $\langle f\rangle^{-1}=-\langle f\rangle$. The unit roots having only real-valued components are called complex structures and represent automorphisms of the tangent fiber bundle $\mathcal{T}\left(M_{n}\right)$ of $M_{n}$. In local frames these appear as particular point-dependent transformations of the gauge group $G(\eta)=\lambda[\mathcal{G}(\eta)]$. The manifold possessing such structures are said to have a Kählerian geometry (see the Appendix A). However, the unit roots considered here are beyond this case since these are defined as automorphisms of the complexified fiber bundle $\mathcal{T}\left(M_{n}\right) \otimes \mathbb{C}$, being thus transformations of the complexified group $G_{c}(\eta)=\lambda\left[\mathcal{G}_{c}(\eta)\right]$.

As in the case of the complex structures of the Kählerian geometries, the matrices of the unit roots have specific algebraic properties resulted from Eq. (22). These can be pointed out in local frames (where the matrix elements are $\left.f_{\cdot \hat{\beta}}^{\hat{\alpha} \cdot}=\hat{e}_{\mu}^{\hat{\alpha}} f_{\cdot \nu}^{\mu \cdot} \cdot e_{\hat{\beta}}^{\nu}\right)$ using gauge transformations of $G(\eta)$.

Lemma 1 The matrix of any root of $M_{n}$ is equivalent with a matrix completely reducible in $2 \times 2$ diagonal blocks.

Proof: The matrix $\langle f\rangle$ which satisfies Eq. (22) has only two-dimensional invariant subspaces spanned by pairs of vectors $z$ and $\langle f\rangle z$. On these subspaces, Eq. (22) is solved in local frames by two types of $2 \times 2$ unimodular blocks without diagonal elements: either skew-symmetric blocks with factors \pm 1 , when the involved dimensions are of the same signature, or symmetric ones with pure imaginary phase factors, $\pm i$, if the signatures are opposite. However, the diagonalization procedure can not be continued using transformations of $G(\eta)$ since these preserve the form of the $2 \times 2$ blocks which are proportional with the generators of the subgroups $S O(2)$ or $S O(1,1)$ acting 
on the corresponding invariant subspaces. Notice that other transformations of $G_{c}(\eta)$ are not useful since these do not leave the metric invariant.

This selects the geometries allowing unit roots.

Corollary 1 The unit roots are allowed only by manifolds $M_{n}$ with an even number of dimensions, $n=2 k, k \leq l$.

Proof: If $n$ is odd then the $2 \times 2$ blocks do not cover all dimensions, so that $\operatorname{det}\langle f\rangle=0$ and $f$ is no more an unit root of $M_{n}$.

Corollary 2 The unit roots of $M_{n}$ have real matrices only when the metric $\eta$ has a signature with even $n_{+}$and $n_{-}$. Otherwise the unit roots have only complex-valued matrices. In both cases the matrices of the unit roots are unimodular, i.e. $\operatorname{det}\langle f\rangle=1$.

It is clear that for the real-valued unit roots (i.e., complex structures) one can construct the symplectic 2 -forms $\tilde{\omega}=\frac{1}{2} f_{\mu \nu} d x^{\mu} \wedge d x^{\nu}$ which are closed and non-degenerate.

The above properties indicate that the unit roots are defined up to sign. Therefore, if two unit roots $f_{1}$ and $f_{2}$ do not obey the condition $f_{1}= \pm f_{2}$ then these will be considered different between themselves. We denote by $\mathcal{R}_{1}\left(M_{n}\right)$ the set of all different unit roots of the manifold $M_{n}$. On the other hand, when an unit root $f$ is multiplied by an arbitrary real number $\alpha \neq 0$, we say that $\xi(x)=\alpha f(x)$ is a root of norm $\|\xi\|=|\alpha|$. Thus we can associate to any unit root $f$ the one-dimensional linear real space $L_{f}=\{\xi \mid \xi=\alpha f, \alpha \in \mathbb{R}\}$ in which each non vanishing element is a root. According to Corollary 2, when the metric $\eta$ is pseudo-Euclidean, the unit roots can have complex matrix elements and in that case the unit root $f$ and its adjoint, $f^{*}$, are different. This last one generates its own linear real space $L_{f^{*}}$ of adjoint roots which satisfy $\left[\langle\xi\rangle^{*},\left\langle\xi^{\prime}\right\rangle\right]=0, \forall \xi, \xi^{\prime} \in L_{f}$ since the matrices of $f$ and $f^{*}$ commutes with each other, having same diagonal blocks up to signs.

The whole set of roots of $M_{n}$ defined as

$$
\mathcal{R}\left(M_{n}\right)=\bigcup_{f \in \mathcal{R}_{1}\left(M_{n}\right)}\left(L_{f}-\{0\}\right)
$$

seems to have special algebraic structure since it does not have the element zero and, in general, it is not certain that a linear combination of roots is a root too. To convince this it is enough to observe that the sum of the roots $\xi$ and $\xi^{*}$ is no more a root since $\operatorname{det}\left(\langle\xi\rangle+\langle\xi\rangle^{*}\right)=0$ when $\xi^{*} \neq \xi$ because of the 
reduction of the pure imaginary diagonal $2 \times 2$ blocks. Moreover, the product of the matrices of two different roots gives a nonsingular matrix but that may be not of a root. Thus we understand that $\mathcal{R}\left(M_{n}\right)+\{0\}$ can not be organized as a global linear space or algebra even though, according to the definition (23), it naturally includes linear parts as $L_{f}$ or $L_{f^{*}}$. In other respects, we know examples indicating that $\mathcal{R}\left(M_{n}\right)$ may contain subsets which are parts of some linear spaces with one or three dimensions, isomorphic with Lie algebras [9, 8]. In any event, the algebraic properties and the topology of $\mathcal{R}\left(M_{n}\right)$ seem to be complicated depending on the topological structure of the set of unit roots $\mathcal{R}_{1}\left(M_{n}\right) \subset \mathcal{R}\left(M_{n}\right)$.

The K-Y tensor gives rise to Dirac-type operators of the form (19). These have an important property formulated in the next theorem [7].

Theorem 1 The Dirac-type operator $D_{f}$ produced by the $K-Y$ tensor $f$ satisfies the condition

$$
\left(D_{f}\right)^{2}=D^{2}
$$

if and only if $f$ is an unit root.

Proof: The arguments of Ref. [7] show that the condition Eq. (24) is equivalent with Eqs. (20) and (21). Moreover, we note that for $f \in \mathcal{R}_{1}\left(M_{n}\right)$ the square of the Dirac-type operator

$$
D_{f}=i f_{\mu}^{\cdot \nu} \cdot \gamma^{\mu} \nabla_{\nu}
$$

has to be calculated exploiting the identity $0=f_{\mu \nu ; \alpha ; \beta}-f_{\mu \nu ; \beta ; \alpha}=f_{\mu \sigma} R_{\cdot \nu \alpha \beta}^{\sigma}+$ $f_{\sigma \nu} R_{\cdot \mu \alpha \beta}^{\sigma}$, which gives $R_{\mu \nu \alpha \beta} f_{\cdot_{\sigma}}^{\mu \cdot} f_{\cdot \tau}^{\nu \cdot}=R_{\sigma \tau \alpha \beta}$ and leads to Eq. (24).

Thus we conclude that the equivalence of the condition (24) with Eqs. (20) and (21) holds in any geometry of dimension $n=2 k$ allowing roots. When $f^{*} \neq f$ then $D_{f^{*}}$ is different from $D_{f}$ even if $\left(D_{f}\right)^{2}=\left(D_{f^{*}}\right)^{2}=D^{2}$. These operators are no longer self-adjoint, $\overline{D_{f}}=D_{f^{*}}$, obey

$$
\left\{D_{f}, D\right\}=0, \quad\left\{D_{f^{*}}, D\right\}=0,
$$

and commute with each other.

\section{Continuous symmetries generated by unit roots}

Thus we arrive at the main point of our study, namely to find the continuous transformations able to relate the operators $D_{f}$ and $D$ to each other. We 
know that in many particular cases [9, 8, 6] this is possible and now we intend to point out that this is a general property of theories involving roots. To this end we introduce a new useful point-dependent matrix.

Definition 2 Given the unit root $f$, the matrix

$$
\Sigma_{f}=\frac{1}{2} f_{\mu \nu} S^{\mu \nu}
$$

is the spin-like operator associated to $f$.

This is an operator that acts on the space of spinors $\Psi^{k}$ and, therefore, can be interpreted as a generator of the spinor representation $\sigma\left[\mathcal{G}_{c}(\eta)\right]$ since the components of $f$ are, in general, complex-valued functions. It has the obvious property $\overline{\Sigma_{f}}=\Sigma_{f^{*}}$ while from (15) and (20) one obtains that it is covariantly constant in the sense that $\nabla_{\nu}\left(\Sigma_{f} \psi\right)=\Sigma_{f} \nabla_{\nu} \psi$. Hereby we find that the Dirac-type operator (25) can be written as

$$
D_{f}=i\left[D, \Sigma_{f}\right]
$$

where $D$ is the standard Dirac operator defined by Eq. (17). Moreover, from Eqs. (26) we deduce $\left[\Sigma_{f}, D^{2}\right]=\left[\Sigma_{f},\left(D_{f}\right)^{2}\right]=0$ and similarly for $\Sigma_{f^{*}}$.

Definition 3 For each $f \in \mathcal{R}_{1}\left(M_{n}\right)$ we define its associated one-parameter Lie group $\mathcal{G}_{f} \subset \mathcal{G}_{c}$ as the group whose operators in the spinor representation are

$$
T(\alpha f)=e^{i \alpha \Sigma_{f}} \in \sigma\left(\mathcal{G}_{f}\right)
$$

where $\alpha \in \mathbb{R}$ is the group parameter.

In other words these are operators of the form (12) where we replace $\omega$ by roots $\xi=\alpha f \in L_{f}$. Consequently, their action on the point-dependent Dirac matrices results from Eq. (13) to be,

$$
T(\alpha f) \gamma^{\mu}[T(\alpha f)]^{-1}=\Lambda_{\cdot \nu}^{\mu \cdot}(\alpha f) \gamma^{\nu},
$$

where $\Lambda_{\cdot \nu}^{\mu \cdot}=e_{\hat{\alpha}}^{\mu} \Lambda_{\cdot{ }_{\hat{\beta}}}^{\hat{\alpha}} \hat{e}_{\nu}^{\hat{\beta}}$ are matrix elements with natural indices of the matrix

$$
\Lambda(\alpha f)=e^{\alpha\langle f\rangle}=\mathbf{I} \cos \alpha+\langle f\rangle \sin \alpha
$$

calculated according to Eqs. (14) and (22). We note that this equation is a matrix representation of the usual Euler formula of the complex numbers. Now it is obvious that in local frames $\langle f\rangle=\Lambda\left(\frac{\pi}{2} f\right) \in \lambda\left(\mathcal{G}_{c}\right)$, as mentioned above. 
Theorem 2 The operators $T(\alpha f) \in \sigma\left(\mathcal{G}_{f}\right)$ have the following action in the space of the operators $D$ and $D_{f}$ :

$$
\begin{aligned}
T(\alpha f) D[T(\alpha f)]^{-1} & =D \cos \alpha-D_{f} \sin \alpha \\
T(\alpha f) D_{f}[T(\alpha f)]^{-1} & =D \sin \alpha+D_{f} \cos \alpha .
\end{aligned}
$$

Proof: From Eq. (31) we obtain the matrix elements $\Lambda_{\cdot \nu}^{\mu \cdot}(\alpha f)=\cos \alpha \delta_{\nu}^{\mu}+$ $\sin \alpha f_{\cdot \nu}^{\mu} \cdot$ which lead to the desired result because $\Sigma_{f}$ is covariantly constant.

From this theorem it results that $\alpha \in[0,2 \pi]$ and, consequently, the group $\mathcal{G}_{f} \sim U(1)$ is compact. Therefore, it must be subgroup of the maximal compact subgroup of $\mathcal{G}_{c}$. In addition, from Eq. (31) we see that $L_{f} \sim s o(2)$ is the Lie algebra of the vector representation of $\mathcal{G}_{f}$ that is the compact group $\lambda\left(\mathcal{G}_{f}\right)=\{\Lambda(\alpha f) \mid \alpha \in[0,2 \pi]\} \sim S O(2)$. Note that the transformations (32) and (33) leave invariant the operator $D^{2}=\left(D_{f}\right)^{2}$ because this commutes with the spin-like operator $\Sigma_{f}$ which generates these transformations.

Particularly, if $M_{n}$ allows real-valued unit roots (i.e. complex structures) this is an usual Kähler manifold. In general, when $f$ has complex components (and $f^{*} \neq f$ ) then $L_{f^{*}} \sim s o(2)$ is a different linear space representing the Lie algebra of $\lambda\left(\mathcal{G}_{f^{*}}\right)$. These two Lie algebras are complex conjugated to each other but remain isomorphic since they are real algebras. The relation among the transformations of $\sigma\left(\mathcal{G}_{f}\right)$ and $\sigma\left(\mathcal{G}_{f^{*}}\right)$ is $\bar{T}(\alpha f)=T\left(-\alpha f^{*}\right)=\left[T\left(\alpha f^{*}\right)\right]^{-1}$ which means that when $f^{*} \neq f$ the representation $\sigma\left(\mathcal{G}_{f}\right)$ is no more unitary in the sense of the generalized Dirac adjoint.

The conclusion is that an unit root produces simultaneously a Dirac-type operator $D_{f}$ which satisfies Eq. (24) and the one-parameter Lie group $\mathcal{G}_{f}$ one needs to relate $D$ and $D_{f}$ to each other.

\section{Symmetries due to families of unit roots}

The next step is to investigate if there could appear higher symmetries given by non-abelian Lie groups with many parameters embedding different abelian groups $\mathcal{G}_{f}$ produced by some sets of unit roots which have to form bases of linear spaces isomorphic with the Lie algebras of these non-abelian groups. Such Lie algebras must include many one-dimensional Lie algebras $L_{f}$ being thus subsets of $\mathcal{R}\left(M_{n}\right)+\{0\}$ where we know that the linear properties are rather exceptions. Therefore, we must look for special families of unit roots, 
$F=\left\{f^{i} \mid i=1,2, \ldots, N_{F}\right\} \subset \mathcal{R}_{1}\left(M_{n}\right)$, having supplementary properties which should guarantee simultaneously that: (I) the linear space $L_{F}=\{\xi \mid \xi=$ $\left.\xi_{i} f^{i}, \xi_{i} \in \mathbb{R}\right\}$ is isomorphic with a real Lie algebra, and (II) each element $\xi \in L_{F}-\{0\}$ is a root (of an arbitrary norm).

The first condition is accomplished only if the set $\left\{T(\xi) \mid \xi \in L_{F}\right\}$ includes a Lie group with $N_{F}$ parameters. This means that the operators $\Sigma^{i}=\Sigma_{f^{i}}, i=1,2, \ldots N_{F}$ must be (up to constant factors) the basis-generators of a Lie algebra with some real structure constants $c_{i j k}$. Then according to Eqs. (11) and (27), we can write

$$
\left[\Sigma^{i}, \Sigma^{j}\right]=\frac{i}{2}\left[\left\langle f^{i}\right\rangle,\left\langle f^{j}\right\rangle\right]_{\mu \nu} S^{\mu \nu}=i c_{i j k} \Sigma^{k}
$$

obtaining a necessary condition for $F$ be a family of unit roots,

$$
\left[\left\langle f^{i}\right\rangle,\left\langle f^{j}\right\rangle\right]=c_{i j k}\left\langle f^{k}\right\rangle
$$

The condition (II) is accomplished only when $\langle\xi\rangle^{2}$ is equal up to a positive factor (i.e. the squared norm) with $-\mathbf{I}$. This requires to have

$$
\left\{\left\langle f^{i}\right\rangle,\left\langle f^{j}\right\rangle\right\}=-2 \kappa_{i j} \mathbf{I}
$$

where $\kappa$ is a positive definite metric that can be brought in canonical form $\kappa_{i j}=\delta_{i j}$ through a suitable choice of the unit roots. If $F$ satisfy simultaneously Eqs. (35) and (36) then $L_{F}$ is just the Lie algebra of the group $\left\{\Lambda(\xi) \mid \xi \in L_{F}\right\}$ the matrices of which read

$$
\Lambda(\xi)=e^{\xi_{i}\left\langle f^{i}\right\rangle}=\mathbf{I} \cos \|\xi\|+\nu_{i}\left\langle f^{i}\right\rangle \sin \|\xi\|
$$

where $\|\xi\|=\sqrt{\xi_{i} \xi_{i}}$ (when we take $\kappa_{i j}=\delta_{i j}$ ) and $\nu_{i}=\xi_{i} /\|\xi\|$. All these results lead to the following theorem.

Theorem 3 If the set $F=\left\{f^{i} \mid i=1,2, \ldots, N_{F}\right\} \in \mathcal{R}_{1}\left(M_{n}\right)$ is a family of unit roots then the matrices $\mathbf{I}$ and $\left\langle f^{i}\right\rangle, i=1,2, \ldots, N_{F}$, form the basis of a matrix representation of a finite-dimensional associative algebra over $\mathbb{R}$.

Proof: If $F$ is a family of unit roots in the sense of above definition then $f^{i}$ must satisfy Eqs. (35) and (36) with canonical metric. Hereby it results that the set of the real linear combinations $\xi_{0} \mathbf{I}+\xi_{i}\left\langle f^{i}\right\rangle$ forms an associative algebra. This algebra is closed with respect to the matrix multiplication that can be calculated by adding the commutator and anticommutator. Moreover 
this algebra is a division one. There exists the zero element (with $\xi_{0}=0, \xi_{i}=$ $0)$, the unit element is $\mathbf{I}$ and each element different from zero has the inverse $\left(\xi_{0} \mathbf{I}+\xi_{i}\left\langle f^{i}\right\rangle\right)^{-1}=\left(\xi_{0} \mathbf{I}-\xi_{i}\left\langle f^{i}\right\rangle\right) /\left(\xi_{0}^{2}+\xi_{i} \xi_{i}\right)$. Obviously, this real algebra is finite possessing a basis of dimension $N_{F}+1$ where $\left\langle f^{i}\right\rangle$ play the role of complex units. Eq. (37) can be interpreted as a matrix representation of the Euler formula.

This theorem severely restricts the existence of the families of unit roots. Indeed, according to the Frobenius theorem there are only two finite real algebras able to give suitable representations in spaces of roots, namely the algebra $\mathbb{C}$ of complex numbers and the quaternion algebra, $\mathbb{H}$. In the first case we have isolated unit roots $f$ and representations of the $\mathbb{C}$ algebra generated by the matrices $\mathbf{I}$ and $\langle f\rangle$ (which play the role of $i \in \mathbb{C}$ ) related to the continuous symmetry group $\mathcal{G}_{f} \sim U(1)$ we studied in the previous section.

Here we focus on the second possibility leading to families of unit roots with $N_{F}=3$ that constitute matrix representations of the quaternion units.

Theorem 4 The unique type of family of unit roots with $N_{F}>1$ having the properties (I) and (II) are the triplets $F=\left\{f^{1}, f^{2}, f^{3}\right\} \subset \mathcal{R}_{1}\left(M_{n}\right)$ which satisfy

$$
\left\langle f^{i}\right\rangle\left\langle f^{j}\right\rangle=-\delta_{i j} \mathbf{I}+\varepsilon_{i j k}\left\langle f^{k}\right\rangle, \quad i, j, k \ldots=1,2,3 .
$$

Proof: Taking into account that $\varepsilon_{i j k}$ is the anti-symmetric tensor with $\varepsilon_{123}=1$ we recognize that Eqs. (38) are the well-known multiplication rules of the quaternion units or similar algebraic structures (e.g. the Pauli matrices). Consequently, the matrices $\left\langle f^{i}\right\rangle$ and $\mathbf{I}$ generate a matrix representation of $\mathbb{H}$. The Frobenius theorem forbids other choices.

If the unit roots $f^{i}$ have only real-valued components we recover the hypercomplex structures defining hyper-Kähler geometries (presented in the Appendix A).

Eqs. (38) combined with the previous results (34)-(37) provide all the features of the specific continuous symmetry associated to $F$. First we observe that the symmetry is governed by the group $\mathcal{G}_{F} \sim S U(2) \subset \mathcal{G}_{c}(\eta)$ whose vector representation is the compact group $\lambda\left(\mathcal{G}_{F}\right)=\left\{\Lambda(\xi) \mid \xi \in L_{F},\|\xi\| \leq 2 \pi\right\}$ formed by the matrices (37) constructed using the elements of the Lie algebra $L_{F} \sim s u(2) \sim s o(3)$.

Corollary 3 The basis-generators of $L_{F}$ are $\frac{i}{2} f^{i}$ while the basis-generators of the su(2) algebra of $\mathcal{G}_{F}$ in spinor representation read $\mathcal{S}^{i}=\frac{1}{2} \Sigma^{i}(i=1,2,3)$. 
Proof: From Eqs. (38) and (35) we deduce that $c_{i j k}=2 \varepsilon_{i j k}$. Furthermore, from Eqs. (34) and (35) we obtain the standard commutation rules of $S U(2)$ generators,

$$
\left[\mathcal{S}^{i}, \mathcal{S}^{j}\right]=i \varepsilon_{i j k} \mathcal{S}^{k}
$$

and similarly for $\frac{i}{2}\left\langle f^{i}\right\rangle$.

We specify that the operators of $\sigma\left(\mathcal{G}_{F}\right)$,

$$
T(\xi)=e^{i \xi_{i} \Sigma^{i}}=e^{2 i \xi_{i} \mathcal{S}^{i}}, \quad \xi=\xi_{i} f^{i} \in L_{F},
$$

can be calculated directly from Eq. (29) replacing $\alpha= \pm\|\xi\|= \pm \sqrt{\xi_{i} \xi_{i}}$ and $f= \pm \xi /\|\xi\|$. Then the transformations the Dirac matrices (30) can be expressed in terms of the parameters $\xi_{i}$ using the matrices $\Lambda(\xi)$ of the form (37). Hereby we observe that $\xi_{i}$ are nothing other than the analogous of the well-known Caley-Klein parameters but ranging in a larger spherical domain (where $\|\xi\| \leq 2 \pi$ ) such that they cover two times the group $\mathcal{G}_{F} \sim S U(2)$.

In the case of triplets involving only real-valued unit roots when the geometry is hyper-Kähler, each family of real unit roots (i.e., a hypercomplex structure) $F$ has its own Lie algebra $L_{F} \sim s u(2)$. These algebras can not be embedded in a larger one because of the restrictions imposed by the Frobenius theorem. An example of hyper-Kähler manifold is the Euclidean Taub-NUT space which is equipped with only one family of real unit roots 6, 9] (see the Appendix B). The manifolds with pseudo-Euclidean metric with odd $n_{+}$ and $n_{-}$have only pairs of adjoint triplets, $F$ and $F^{*}$, the last one being formed by the adjoints of the unit roots of $F$. The spaces $L_{F}$ and $L_{F^{*}}$ are isomorphic between themselves (as real vector spaces) and all the results concerning the symmetries generated by $F^{*}$ can be taken over from those of $F$ using complex conjugation. Moreover, we must specify that the set $L_{F} \cup L_{F^{*}}$ is no more a linear space since the linear operations among the elements of $L_{F}$ and $L_{F^{*}}$ are not allowed. A typical example is the Minkowski spacetime which has a pair of conjugated triplets of complex-valued unit roots [8] (presented in Appendix C). Both these examples of manifolds possessing triplets with the properties (38) are of dimension four. As we know, the results indicate that similar properties could have all the flat manifolds of dimension $n=4 k, k=1,2,3, \ldots$ where we expect to find many such triplets 15.

Starting with a triplet $F=\left\{f^{1}, f^{2}, f^{3}\right\} \subset \mathcal{R}_{1}\left(M_{n}\right)$ with the properties (38) one can construct a rich set of Dirac-type operators of the form $D_{\nu}=$ $\nu_{i} D^{i}$ where $\nu$ is an unit vector $\left(\right.$ with $\nu_{i} \nu_{i}=1$ ) and $D^{i}=D_{f^{i}}=i\left[D, \Sigma^{i}\right]$, 
$i=1,2,3$, play the role of a basis. This set is compact and isomorphic with the sphere of unit roots $\mathbf{S}_{F}=\left\{f_{\nu} \mid f_{\nu}=\nu_{i} f^{i}, \nu^{2}=1\right\} \subset L_{F}$, since $D_{\nu}=D_{f_{\nu}}$ for any $f_{\nu} \in \mathbf{S}_{F}$. Each operator $D_{\nu}$ can be related to $D$ through the transformations (32) and (33) of the spinor representation of the oneparameter subgroup $\mathcal{G}_{f_{\nu}} \subset \mathcal{G}_{F} \sim S U(2)$ defined by $f_{\nu}$. The general $S U(2)$ transformations in the basis $D, D^{i}(i=1,2,3)$ read

$$
\begin{aligned}
T(\xi) D[T(\xi)]^{-1} & =D \cos \|\xi\|-\nu_{i} D^{i} \sin \|\xi\|, \\
T(\xi) D^{i}[T(\xi)]^{-1} & =D^{i} \cos \|\xi\|+\left(\nu_{i} D+\varepsilon_{i j k} \nu_{j} D^{k}\right) \sin \|\xi\|,
\end{aligned}
$$

where $\xi=\|\xi\| \nu_{i} f^{i}$.

The basis operators $D^{i}$ anticommute with $D$ and, consequently, all the Dirac-type operators $D_{\nu}$ anticommute with $D$. Moreover, the next theorem extends this property up to a $N=4$ superalgebra.

Theorem 5 If a triplet $F \subset \mathcal{R}_{1}\left(M_{n}\right)$ accomplishes Eqs. (38) then the corresponding Dirac-type operators satisfy the superalgebra

$$
\left\{D^{i}, D^{j}\right\}=2 \delta_{i j} D^{2} \text {. }
$$

Proof: If $i=j$ we take over the result of Theorem 11. For $i \neq j$ the concrete calculation shows that $D^{i}$ and $D^{j}$ anticommute.

Thus it is clear that the operators $D$ and $D^{i}(i=1,2,3)$ form a superalgebra with four supercharges [6]. Moreover, Eqs. (43) remain invariant under transformations (41) and (42) since $T(\xi)$ and $D^{2}$ commute between themselves.

The previous results indicate that the set $\mathcal{R}_{1}\left(M_{n}\right)$, of unit roots producing Dirac-type operators, has an interesting topological structure involving either single $f$ producing isolated Dirac-type operators or unit spheres $\mathbf{S}_{F}$ leading to compact sets of Dirac-type operators. In order to show off this structure one needs to correctly identify all the independent triplets with the properties (38) or the different singles. To this end one has to exploit the mechanisms of our theory based on the fact that the linear spaces $L_{f}$ or $L_{F}$ are isomorphic with the Lie algebras of the symmetry groups of the Dirac-type operators generated by spin-like operators.

\section{Discrete symmetries}

In the case when there appear difficulties because of a large number of different singles or triplets, the study of the discrete symmetries could be also 
productive. Of course, the results concerning the continuous symmetries obtained above will be crucial for understanding the structure of the discrete transformations which relate among themselves the standard Dirac operator and the Dirac-type ones.

Let us start with the simplest case of an isolated unit root $f$.

Theorem 6 For any unit root $f$ there exists the discrete group $\mathbb{Z}_{4}(f) \subset \mathcal{G}_{f}$ the orbit of which is $\left\{D,-D, D_{f},-D_{f}\right\}$.

Proof: Using Eqs. (32) and (33) one observes that the transformations $I$, $U_{f}=T\left(-\frac{\pi}{2} f\right), P=\left(U_{f}\right)^{2}=T(\pi f)$, and $\left(U_{f}\right)^{3}=T\left(\frac{\pi}{2} f\right)=P U_{f}=U_{f} P$ form the spinor representation of the cyclic group $\mathbb{Z}_{4}(f)$. Since $P^{2}=I$, the pair $(I, P)$ represents the subgroup $\mathbb{Z}_{2} \subset \mathbb{Z}_{4}(f)$. According to Eq. (32) we find that

$$
D_{f}=U_{f} D\left(U_{f}\right)^{-1}
$$

and the action of the operator $P$,

$$
P \gamma^{\mu} P=-\gamma^{\mu}
$$

is independent on the form of $f$ so that this changes the sign of all the Dirac or Dirac-type operators.

For a given manifold, $M_{n}$, the operator $P$ is uniquely defined up to a factor $N$ which satisfies $N^{2}=I$ and commutes with all the operators of the spin theory on $M_{n}$. In examples we know this is $N= \pm I$ (see the Appendix C). Thus $P$ is in some sense independent on the discrete symmetry group where is involved, playing a similar role as the matrix $\gamma^{5}$ in the usual theory of the Dirac spinors.

When the metric is pseudo-Euclidean then the operators of the spinor representation of the discrete group $\mathbb{Z}_{4}\left(f^{*}\right)$ produced by $f^{*} \neq f$ have to be written directly using the Dirac adjoint. From Eq. (444) we obtain

$$
U_{f^{*}}=\left(\overline{U_{f}}\right)^{-1}=\overline{U_{f}} \bar{P}
$$

where, as observed, $\bar{P}= \pm P$. One can show that the sign is $(+)$ when $\gamma$ is built from an even number of gamma matrices and $(-)$ when this number is odd. Starting with these elements the remaining operators of the cyclic group will be obtained using multiplication.

A most interesting case is that of the discrete symmetries of the Diractype operators $D^{i}(i=1,2,3)$ given by the triplet $F$ which satisfy Eqs. (38). 
Theorem 7 The Dirac operators $\pm D$ and the Dirac-type ones $\pm D^{1}, \pm D^{2}$, and $\pm D^{3}$ are related among themselves through the transformations of the spinor representation of the quaternion group, $\mathbb{Q}(F) \subset \mathcal{G}_{F}$.

Proof: Let us denote by $U_{i}=T\left(-\frac{\pi}{2} f^{i}\right)$ the operators that, according to Theorem [6] have the properties

$$
\left(U_{1}\right)^{2}=\left(U_{2}\right)^{2}=\left(U_{3}\right)^{2}=P, \quad P^{2}=I,
$$

and $P U_{i}=U_{i} P$. Furthermore, from Eqs. (30), (31) and (38) we deduce

$$
\begin{aligned}
& U_{1} U_{2}=U_{3}, \quad U_{2} U_{3}=U_{1}, \quad U_{3} U_{1}=U_{2}, \\
& U_{2} U_{1}=U_{3} P, \quad U_{3} U_{2}=U_{1} P, \quad U_{1} U_{3}=U_{2} P,
\end{aligned}
$$

and, after a few manipulation, we see that $I, P, U_{i}$ and $P U_{i}(i=1,2,3)$ form a representation of the dicyclic group $\langle 2,2,2\rangle$ which is isomorphic with the quaternion subgroup of $\mathcal{G}_{F}$ we denote by $\mathbb{Q}(F)$. Using Eqs. (44) and (45) we find that its orbit in the space of the Dirac operators is the desired one.

As expected, the cyclic groups $\mathbb{Z}_{4}\left(f^{i}\right)$ are subgroups of $\mathbb{Q}(F)$. For this reason the spinor representation of the group $\mathbb{Q}\left(F^{*}\right)$ has to be derived from that of $\mathbb{Q}(F)$ using the same method as in the case of cyclic groups.

Hence we conclude that for each isolated unit root $f$ one can define a finite group $\mathbb{Z}_{4}(f)$ which is a subgroup of $\mathcal{G}_{f}$ while the triplets $F$ produce more complicated finite discrete groups, $\mathbb{Q}(F) \subset \mathcal{G}_{F}$. Since the groups $\mathcal{G}_{f}$ and $\mathcal{G}_{F}$ can not be embedded in a larger group, the product of two operators of the spinor representation of two different discrete groups is, in general, an arbitrary operator which do not correspond to a transformation of another discrete group $\mathbb{Z}_{4}$ or $\mathbb{Q}$. In addition, this new operator could transform the standard Dirac operator in a new operator having different properties to those of the Dirac-type ones. Therefore when we restrict ourselves to orbits containing only the Dirac and Dirac-type operators we have to consider only the discrete groups discussed above.

\section{Concluding remarks}

Here we have studied the symmetries and supersymmetries of the Dirac-type operators built with the help of the unit roots which in general do not accomplish the complex structures of the Kählerian geometries. This extension, 
that seems to be pointless in geometry and classical physics, is very useful from the point of view of the Dirac theory since it permits to construct Diractype operators even in geometries which do not admit complex structures, as in the case of the Minkowski spacetime. Therefore, now it is certain that isolated or triplets of Dirac-type operators arise in any flat manifold of dimension $n=2 k$ indifferent on the metric signature. Of course, the triplets of Dirac-type operators produced by families of unit roots could appear only in manifolds of dimensions $n=4 k$.

On the other hand, the use of the roots in manifold of arbitrary dimensions brings nothing new from the point of view of the symmetry and supersymmetry of the Dirac-type operators since these can not be larger than those we have found, for example, in the Euclidean Taub-NUT space that is a hyper-Kähler manifold [6, 9]. Thus in any concrete theory we expect to find at most continuous $S U(2)$ symmetries coupled to quaternionic discrete ones associated to the $N=4$ superalgebras of Dirac and Dirac-type operators. What is remarkable is that there are geometries with many such superalgebras that can not be embedded in larger one showing off a higher symmetry. This is a new phenomenon which may have consequences not only in the Dirac theory but in geometry too. We hope that our method could be a good tool for investigating delicate problems concerning the topology of these rich sets of Dirac-type operators.

Technically speaking, the important results we obtained here are the rule (28) of generating Dirac-type operators and the definitive form of the transformations (41) and (42) of the representation $\sigma\left(\mathcal{G}_{F}\right)$. In both cases the main role is played by the spin-like operators $\Sigma^{i}$ which simultaneously give rise to the Dirac-type operators and define the generators of these transformations. However, this fact is not surprising since one knows that they have pseudo-classical analogues with an interesting behavior.

In the pseudo-classical spinning particle models in curved spaces from covariantly constant $\mathrm{K}-\mathrm{Y}$ tensors $f_{\mu \nu}$ can be constructed conserved quantities of the type $f_{\mu \nu} \theta^{\mu} \theta^{\nu}$ depending on the Grassmann variables $\left\{\theta^{\mu}\right\}$ [16. The Grassmann variables $\left\{\theta^{\mu}\right\}$ transform as a tangent space vector and describe the spin of the particle. The antisymmetric tensor $S^{\mu \nu}=-i \theta^{\mu} \theta^{\nu}$ generates the internal part of the local tangent space rotations. For example, in the spinning Euclidean Taub-NUT space such operators correspond to components of the spin which are separately conserved [17.

The construction of the new supersymmetries in the context of pseudoclassical mechanics can be carried over straightforwardly to the case of quan- 
tum mechanics by the usual replacement of phase space coordinates by operators and Poisson-Dirac brackets by anticommutators [18. In terms of these operators the supercharges are replaced by Dirac-type operators [19. In both cases, the correspondence principle leads to equivalent algebraic structures making obvious the relations between these approaches [17].

It is worth to mention the role of non-covariantly constant $\mathrm{K}-\mathrm{Y}$ tensors in generating hidden symmetries. The presence of non-covariantly constant K$\mathrm{Y}$ tensors implies the existence of non-standard supersymmetries in point particle theories on curved background. In general, in such a case, the Stäckel-Killing tensor $K_{\mu \nu}$ in Eq. (11) is not covariantly constant and the corresponding non-standard supercharges $Q_{a}$ of hidden supersymmetries do not close on the Hamiltonian [16] (or on $D^{2}$, as in standard Dirac theories), but $\left\{Q_{a}, Q_{b}\right\} \propto J \delta_{a b}$, where $J$ is an invariant different from the Hamiltonian (or $D^{2}$ ).

The Dirac type operators associated with the non-covariantly constant $\mathrm{K}-\mathrm{Y}$ tensors were investigated in the case of the hidden symmetries of the Euclidean Taub-NUT space [6]. A more systematic study of the symmetries generated by the non-covariantly constant K-Y tensors and the properties of the corresponding Dirac operators will be given elsewhere.

\section{Acknowledgments}

The authors would like to express their gratitude to the anonymous referees for comments and suggestions that have improved this article.

\section{Appendix A Kählerian geometries}

Let us consider the manifold $M_{n}(n=2 k)$ and its tangent fiber bundle, $\mathcal{T}\left(M_{n}\right)$, assuming that $M_{n}$ is equipped with a complex structure that is a particular bundle automorphism $h: \mathcal{T}\left(M_{n}\right) \rightarrow \mathcal{T}\left(M_{n}\right)$ which satisfies $\langle h\rangle^{2}=$ -I and is covariantly constant. Notice that the matrix of $h$ in local frames is an orthogonal point-dependent transformation of the gauge group $G(\eta)$. With its help one gives the following definition [20, 15]:

Definition 4 A Riemannian metric $g$ on $M_{n}$ is said Kählerian if $h$ is pointwise orthogonal, i.e., $g(h X, h Y)=g(X, Y)$ for all $X, Y \in \mathcal{T}_{x}\left(M_{n}\right)$ at all 
points $x$.

In local coordinates, $h$ is a skew-symmetric second rank tensor with realvalued components, $h_{\mu \nu}=-h_{\nu \mu}$, which obey $g_{\mu \nu} h_{\cdot \alpha}^{\mu \cdot} h_{\cdot \dot{\beta}}^{\nu}=g_{\alpha \beta}$. This gives rise to the symplectic form $\tilde{\omega}=\frac{1}{2} h_{\nu \mu} d x^{\nu} \wedge d x^{\mu}$ (i.e., closed and non-degenerate). Alternative definitions can be formulated starting with both, $g$ and $\tilde{\omega}$, which have to satisfy the Kähler relation $\tilde{\omega}(X, Y)=g(X, h Y)$ [15].

A hypercomplex structure on $M_{n}$ is an ordered triplet $H=\left(h^{1}, h^{2}, h^{3}\right)$ of complex structures on $M_{n}$ satisfying Eq. (38). In Lie algebraic terms, the matrices $\frac{1}{2}\left\langle h^{j}\right\rangle$ realize the $s u(2)$ algebra.

Definition 5 A hyper-Kähler manifold is a manifold whose Riemannian metric is Kählerian with respect to each different complex structures $h^{1}, h^{2}$ and $h^{3}$.

Our unit roots, $f$, are defined in a similar way as the complex structures with the difference that the unit roots are automorphisms of the complexified tangent bundle, $f: \mathcal{T}\left(M_{n}\right) \otimes \mathbb{C} \rightarrow \mathcal{T}\left(M_{n}\right) \otimes \mathbb{C}$. Therefore, $f$ have complexvalued components and the transformation matrix $\langle f\rangle$ is of the complexified group $G_{c}(\eta)$. Thus it is clear that the real-valued unit roots are complex structures as defined above. The families of unit roots may differ from the hypercomplex structures but have the same algebraic properties given by Ec. (38).

The passing from the complex structures to unit roots is productive from the point of view of the Dirac theory since in this way one can introduce families of unit roots generating superalgebras of Dirac-type operators even in manifolds which do not admit complex structures. The Minkowski spacetime is a typical example. This will be briefly presented after discussing the Euclidean Taub-NUT space which is hyper-Kähler [21, 22].

\section{Appendix B}

\section{Example: Euclidean Taub-NUT space}

In the spacetime of the Kaluza-Klein theory, $M_{(1,4)}$, corresponding to the usual Dirac spinor space, the Euclidean Taub-NUT space, $M_{(, 4)}$, covers the whole space part which is of dimension four. We take the metric $\eta=$ $\operatorname{diag}(-1,-1,-1,-1)$ and a chart with Cartesian coordinates $x^{\mu}(\mu, \nu, \ldots=$ 
$1,2,3,5)$ where the line element reads

$$
d s^{2}=g_{\mu \nu} d x^{\mu} d x^{\nu}=-\frac{1}{V} d l^{2}-V\left(d x^{5}+A_{i} d x^{i}\right)^{2} .
$$

The notation $d l^{2}=(d \vec{x})^{2}$ stands for the Euclidean three-dimensional line element and $\vec{A}$ is the gauge field of a monopole. Another chart suitable for applications is of spherical coordinates, $(r, \theta, \phi, \chi)$, among them the first three are the spherical coordinates associated with the Cartesian space ones, $x^{i}(i, j, \ldots=1,2,3)$, and $\chi+\phi=-x^{5} / \mu$. The real number $\mu$ is the parameter defining the function $1 / V(r)=1+\mu / r$ while the unique non-vanishing component of the vector potential in spherical coordinates is $A_{\phi}=\mu(1-\cos \theta)$.

Furthermore, we consider the local frames given by tetrad fields $e(x)$ and $\hat{e}(x)$ as defined in 23 while the four Dirac matrices $\gamma^{\hat{\alpha}}$, that satisfy $\left\{\gamma^{\hat{\alpha}}, \gamma^{\hat{\beta}}\right\}=-2 \delta^{\hat{\alpha} \hat{\beta}}$, have to be written in the following representation

$$
\gamma^{i}=\left(\begin{array}{cc}
0 & \sigma_{i} \\
-\sigma_{i} & 0
\end{array}\right), \quad \gamma^{5}=i\left(\begin{array}{cc}
0 & \mathbf{1}_{2} \\
\mathbf{1}_{2} & 0
\end{array}\right) .
$$

In addition we consider the matrix $\gamma^{0}=\gamma^{1} \gamma^{2} \gamma^{3} \gamma^{5}=\operatorname{diag}\left(\mathbf{1}_{2},-\mathbf{1}_{2}\right)$ which is involved in the Kaluza-Klein theory of The Dirac equation explicitly depending on time [5].

The standard Dirac operator of the theory without explicit mass term is defined in local frames as $D=i \gamma^{\hat{\alpha}} \nabla_{\hat{\alpha}}$ [5, 9] where the spin covariant derivatives with local indices, $\nabla_{\hat{\alpha}}$, depend on the momentum operators, $P_{i}=$ $-i\left(\partial_{i}-A_{i} \partial_{5}\right)$ and $P_{5}=-i \partial_{5}$, and spin connection [5], such that the Dirac operator

$$
D=\left(\begin{array}{cc}
0 & D^{(+)} \\
D^{(-)} & 0
\end{array}\right)
$$

can be expressed in terms of Pauli operators,

$$
D^{(-)}=\sqrt{V}\left(-\vec{\sigma} \cdot \vec{P}+\frac{i P_{5}}{V}\right), \quad D^{(+)}=V\left(\vec{\sigma} \cdot \vec{P}+\frac{i P_{5}}{V}\right) \frac{1}{\sqrt{V}},
$$

involving the Pauli matrices, $\sigma_{i}$. Notice that the Klein-Gordon operator of the Euclidean Taub-NUT space $\Delta=\nabla_{\mu} g^{\mu \nu} \nabla_{\nu}=-D^{(+)} D^{(-)}$commutes with $\sigma_{i}$ but $D^{(-)} D^{(+)}$does not have this property because of its complicated form involving spin terms [5, []. 
For this reason there is only one triplet of unit roots, $F=\left\{f^{(1)}, f^{(2)}, f^{(3)}\right\}$, which satisfy Ec. (38). Their non-vanishing components in the local frame are real,

$$
\begin{array}{lll}
\hat{f}_{23}^{(1)}=1, & \hat{f}_{31}^{(2)}=1, & \hat{f}_{12}^{(3)}=1, \\
\hat{f}_{51}^{(1)}=1, & \hat{f}_{52}^{(2)}=1, & \hat{f}_{53}^{(3)}=1,
\end{array}
$$

such that they form a hypercomplex structure and the Euclidean Taub-NUT space is hyper-Kähler. They give rise to the spin-like operators

$$
\Sigma^{(i)}=\frac{i}{4} \hat{f}_{\hat{\alpha} \hat{\beta}}^{(i)} \gamma^{\hat{\alpha}} \gamma^{\hat{\beta}}=\left(\begin{array}{cc}
\sigma_{i} & 0 \\
0 & 0
\end{array}\right)
$$

and, according to Eq. (28), produce the Dirac-type operators [5]

$$
D^{(i)}=i\left[D, \Sigma^{(i)}\right]=\left(\begin{array}{cc}
0 & -i \sigma_{i} D^{(+)} \\
i D^{(-)} \sigma_{i} & 0
\end{array}\right)
$$

which anticommute with $D$ and $\gamma^{0}$.

The $S U(2)$ transformations (40) generated by the above defined spin-like operators are

$$
T(\vec{\xi})=e^{i \vec{\xi} \cdot \vec{\Sigma}}=\left(\begin{array}{cc}
\hat{U}(\vec{\xi}) & 0 \\
0 & \mathbf{1}_{2}
\end{array}\right),
$$

where $\vec{\xi}=\alpha \vec{\nu}\left(\vec{\nu}^{2}=1\right)$ and $U$ is the $S U(2)$ transformation

$$
\hat{U}(\vec{\xi})=e^{i \vec{\xi} \cdot \vec{\sigma}}=\mathbf{1}_{2} \cos \alpha+i \vec{\nu} \cdot \vec{\sigma} \sin \alpha .
$$

The last step is to write down the final form of the concrete transformations according to Eqs. (41) and (42).

The discrete transformations of the group $\mathbb{Q}(F)$ are $I, P=\operatorname{diag}\left(-\mathbf{1}_{2}, \mathbf{1}_{2}\right)$ and the sets of matrices $U_{(k)}=\operatorname{diag}\left(-i \sigma_{k}, \mathbf{1}_{2}\right)$ and $P U_{(k)}$.

In the Euclidean Taub-NUT space, in addition to the above discussed complex structure, there exists a fourth Killing-Yano tensor,

$$
f_{\alpha \beta}^{Y}=-\frac{x^{i}}{r} f_{\alpha \beta}^{(i)}+\frac{2 x^{i}}{\mu V} \varepsilon_{i j k} \hat{e}_{\alpha}^{j} \hat{e}_{\beta}^{k},
$$

which is not covariantly constant. The presence of $f^{Y}$ is due to the existence of the hidden symmetries of the Euclidean Taub-NUT geometry which are encapsulated in three non-trivial Stäckel-Killing tensors. These are interpreted as the components of the so-called Runge-Lenz vector of the Euclidean TaubNUT problem and are expressed as symmetrized products of the Killing-Yano tensors $f^{Y}$ and $f^{(i)},(i=1,2,3)[6]$. 


\section{Appendix $\mathrm{C}$}

\section{Example: Minkowski spacetime}

In the Minkowski spacetime, $M_{(1,3)} \subset M_{(1,4)}$, with metric $\eta=(1,-1,-1,-1)$ we take the gauge $e_{\nu}^{\mu}=\hat{e}_{\nu}^{\mu}=\delta_{\nu}^{\mu},(\mu, \nu, \ldots=0,1,2,3)$. We use the chiral representation of the Dirac matrices where the standard Dirac operator reads

$$
D=i \gamma^{\mu} \partial_{\mu}=\left(\begin{array}{cc}
0 & i\left(\partial_{t}+\vec{\sigma} \cdot \vec{\partial}\right) \\
i\left(\partial_{t}-\vec{\sigma} \cdot \vec{\partial}\right) & 0
\end{array}\right)=\left(\begin{array}{cc}
0 & D^{(+)} \\
D^{(-)} & 0
\end{array}\right)
$$

and the generators of the spinor representation of the group $\mathcal{G}(\eta)=S L(2, \mathbb{C})$ take the form $S^{i j}=\frac{1}{2} \varepsilon_{i j k} \operatorname{diag}\left(\sigma_{k}, \sigma_{k}\right)$ and $S^{i 0}=\frac{i}{2} \operatorname{diag}\left(\sigma_{i},-\sigma_{i}\right)$.

There are two families of three roots [8]. The unit roots of the first triplet, $F$, have the non-vanishing complex components [8]

$$
\begin{aligned}
f_{23}^{(1)}=1, & f_{31}^{(2)}=1, & f_{12}^{(3)}=1, \\
f_{01}^{(1)}=i, & f_{02}^{(2)}=i, & f_{03}^{(3)}=i,
\end{aligned}
$$

and, consequently, $F$ is not a hypercomplex structure and the Minkowski spacetime is not a hyper-Kähler manifold. The corresponding spin-like operators

$$
\Sigma^{(i)}=\frac{1}{2} f_{\mu \nu}^{(i)} S^{\mu \nu}=\left(\begin{array}{cc}
\sigma_{i} & 0 \\
0 & 0
\end{array}\right)
$$

give Dirac-type operators $D^{(i)}=i\left[D, \Sigma^{(i)}\right]$ of the same form as (B.8) and the generators $\mathcal{S}^{(i)}=\frac{1}{2} \Sigma^{(i)}$ of the spinor representation of the group $\mathcal{G}_{F}=S U(2)$. The second triplet is $F^{*}$ for which all the spinor quantities are just the Dirac conjugated of those of $F$ (using $\gamma=\gamma^{0}$ ). The corresponding spinlike operators are

$$
\overline{\Sigma^{(i)}}=\frac{1}{2}\left(f_{\mu \nu}^{(i)}\right)^{*} S^{\mu \nu}=\left(\begin{array}{cc}
0 & 0 \\
0 & \sigma_{i}
\end{array}\right)
$$

which means that the $S U(2)$ groups $\sigma\left(\mathcal{G}_{F}\right)$ and $\sigma\left(\mathcal{G}_{F^{*}}\right)$ act separately on the left and right-handed parts of the Dirac spinor. Moreover, it is interesting to observe that $\Sigma^{(i)}+\overline{\Sigma^{(i)}}=2 S^{i}$ where $S^{i}=\frac{1}{2} \varepsilon_{i j k} S^{j k}$ are the usual spin operators. This perfect balance between the chiral sectors is due to the fact that the operator $D^{(+)} D^{(-)}=D^{(-)} D^{(+)}$commutes with $\sigma_{i}$.

The discrete symmetry is given by two representations of the quaternion group acting on each of both chiral sectors. On the left-handed sector 
acts the group $\mathbb{Q}(F)$ represented by the operators $I, P=\operatorname{diag}\left(-\mathbf{1}_{2}, \mathbf{1}_{2}\right)$, $U_{(i)}=\operatorname{diag}\left(-i \sigma_{i}, \mathbf{1}_{2}\right)$ and $P U_{(i)}$. The operators of the group of the righthanded sector, $\mathbb{Q}\left(F^{*}\right)$, can be obtained using the Dirac adjoint and taking into account that here $\bar{P}=-P$. The resulted operators, $\operatorname{diag}\left(\mathbf{1}_{2}, \pm i \sigma_{i}\right)$, act only on the right-handed sector.

Hence it is clear that each chiral sector has its own sets of unit roots defining Dirac-type operators. These are the spheres $\mathbf{S}_{F}$ of the left-handed sector and $\mathbf{S}_{F^{*}}$ of the right-handed one. Since there are no other independent unit roots, the whole set of unit roots of the Minkowski spacetime is $\mathcal{R}_{1}(M)=$ $\mathbf{S}_{F} \cup \mathbf{S}_{F^{*}}$. The continuous and discrete symmetry groups of the Dirac-type operators are defined separately on each of these two spheres. The continuous one is governed by two separated Lie algebras, $L_{F} \sim s o(3)$ (for the lefthanded sector) and $L_{F^{*}} \sim s o(3)$ (for the right-handed one), rather than by a total Lie algebra $s o(3) \oplus s o(3) \sim s o(4)[8$.

\section{References}

[1] K. Yano, Ann. Math. 55 (1952) 328.

[2] G. W. Gibbons, R. H. Rietdijk and J. W. van Holten, Nucl. Phys. B404 (1993) 42.

[3] B. Carter and R. G. McLenaghan, Phys. Rev. D 19 (1979) 1093.

[4] R. Penrose, Ann. NY Acad. Sci. 224 (1973) 125; R. Floyd, The dynamics of Kerr fields, (Ph D. Thesis, London, 1973).

[5] I. I. Cotăescu and M. Visinescu, hep-th/0008181, Int. J. Mod. Phys. A 16 (2001) 1743.

[6] I. I. Cotăescu and M. Visinescu, hep-th/0101163, Phys. Lett. B502 (2001) 229; hep-th/0102083, Class. Quantum Grav. 18 (2001) 3383; hep-th/0107205, J. Math. Phys. 43 (2002) 2978.

[7] V. V. Klishevich, Class. Quantum Grav. 17 (2000) 305.

[8] V. V. Klishevich, Class. Quantum Grav. 19 (2002) 4287.

[9] I. I. Cotăescu and M. Visinescu, hep-th/0202034, Gen. Relat. Gravit. 35 (2003) 389. 
[10] N. Salingaros, J. Math. Phys 23 (1982) 1.

[11] A. Van Proeyen, hep-th/9910030, Annals of the University of Craiova, Physics AUC 9 (1999) 1.

[12] I. I. Cotăescu, J. Phys. A: Math. Gen. 33 (2000) 9177.

[13] R. G. McLenaghan and Ph. Spindel, Phys. Rev. D 20 (1979) 409.

[14] N. Kamran and R. G. McLenaghan, Phys. Rev. D 30 (1984) 357.

[15] G. Gaeta and P. Morando, math-ph/0204019, J. Phys. A: Math. Gen. 35 (2002) 3925.

[16] D. Vaman and M. Visinescu, Phys. Rev. D 57 (1998) 3790.

[17] J. W. van Holten, Phys. Lett. B 342 (1995) 47.

[18] F. A. Berezin and M. S. Marinov, Ann. Phys. (N. Y.) 104 (1977) 336.

[19] R. H. Rietdijk, Applications of supersymmetric quantum mechanics, PhD. Thesis, Amsterdam (1992); J. W. van Holten, gr-qc/9910035, Annalen Phys. 9SI (2000) 83.

[20] H. B. Lawson, Jr. and M.-L. Michelsohn, Spin Geometry, (Princeton Univ. Press, 1989).

[21] M. Atiyah and N. Hitchin, The geometry and dynamics of magnetic monopoles, (Princeton Univ. Press, 1988).

[22] G. W. Gibbons and P. J. Ruback, Commun. Math. Phys. 115 (1988) 267.

[23] H. Boutaleb - Joutei and A. Chakrabarti, Phys. Rev. D21 (1979) 2280. 